\title{
Absolute and differential measurement of water vapor supersaturation using a commercial thin-film sensor
}

\author{
C. K. Chng, H. Yu, and K. G. Libbrecht ${ }^{a)}$ \\ Norman Bridge Laboratory of Physics, California Institute of Technology 264-33, Pasadena, \\ California 91125
}

(Received 2 September 1999; accepted for publication 17 September 1999)

\begin{abstract}
We describe a technique for measuring the water vapor supersaturation of normal air over a temperature range of $-40 \leqslant T \leqslant 0{ }^{\circ} \mathrm{C}$. The measurements use an inexpensive commercial hygrometer which is based on a thin-film capacitive sensor. The time required for the sensor to reach equilibrium was found to increase exponentially with decreasing sensor temperature, exceeding 2 $\min$ for $T=-30{ }^{\circ} \mathrm{C}$; however, the water vapor sensitivity of the device remained high down to this temperature. After calibrating our measurement procedure, we found residual scatter in the data corresponding to an uncertainty in the absolute water vapor pressure of about $\pm 15 \%$. This scatter was due mainly to long-term drift, which appeared to be intrinsic to the capacitive thin-film sensor. The origin of this drift is not clear, but it effectively limits the applicability of this instrument for absolute measurements. We also found, however, that the high sensitivity of the thin-film sensor makes it rather well suited for differential measurements. By comparing supersaturated and saturated air at the same temperature we obtained a relative measurement uncertainty of about $\pm 1.5 \%$, an order of magnitude better than the absolute measurements. (C) 2000 American Institute of Physics. [S0034-6748(00)04701-8]
\end{abstract}

\section{INTRODUCTION}

Our desire to obtain simple and accurate measurements of the water vapor supersaturation of air is motivated by our study of the crystal growth of ice from the vapor phase. Although this is an extremely common meteorological phenomenon (resulting in the precipitation of snow crystals in the atmosphere), the physics of ice crystal growth is very poorly understood. ${ }^{1}$ The morphology of ice grown from the vapor phase is dominated by two physical effects-water vapor diffusion and ice surface kinetics-and the theoretical machinery to disentangle these two effects in realistic circumstances has only recently begun to appear. ${ }^{2,3}$ Laboratory measurements then reveal that the surface kinetics of ice crystal growth exhibit a remarkably complex dependence on temperature. ${ }^{1,4,5}$ There has been considerable speculation as to the underlying physical mechanisms responsible for this behavior, and it is widely believed that the presence of a disordered (or premelted) layer on the ice surface plays a significant role in the surface kinetics. ${ }^{1,6,7}$ Understanding how a disordered layer in general affects crystal growth dynamics remains an active research area.

The ice growth rates depend on water vapor supersaturation as well as temperature, and measurements over a broad range of temperatures, supersaturations, as well as in different background gases, are likely to play a substantial role in realizing an effective model of ice crystal growth. While temperature and gas pressure are easily measured in an ice crystal growth chamber, supersaturation is more difficult, partly because of diffusion and convection in such chambers, along with the boundary conditions at the walls or

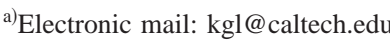

wherever there is an ice surface. In particular, a stationary water vapor sensor cannot be placed in a supersaturated chamber, since the sensor will quickly become coated with ice and thus itself affect the local supersaturation level.

Two solutions to the supersaturation measurement problem have been used previously. The first is to calculate the supersaturation directly, for example, by mixing known quantities of saturated air at different temperatures, ${ }^{8}$ by modeling vapor transport in a diffusion chamber, ${ }^{9}$ or by using a supercooled water mixture. ${ }^{10}$ These techniques have some potential for error if the experimental system is not extremely well characterized.

A second solution is to extract a sample of supersaturated air, and subsequently measure the amount of water contained in the air. ${ }^{11,12} \mathrm{~A}$ variation of this solution, which is used here, is to extract a small volume of supersaturated air, warm it to above the dew/frost point, and then measure the relative humidity. Plunging a small warm extraction tube into a supersaturated chamber produces only a minimal perturbation to the supersaturation level, provided a small volume is extracted quickly. Knowing the relative humidity, sensor temperature, and the initial air temperature, the original supersaturation level can in principle be obtained, assuming no water is lost or added to the sampled air in processing.

Common methods used for relative humidity measurements include chilled mirror hygrometers and thin-film capacitive sensors, and for both of these there are a variety of commercial units available. The absolute accuracy of commercial chilled mirror hygrometers can be as high as $\pm 2{ }^{\circ} \mathrm{C}$ in the measurement of the dew/frost point, ${ }^{13,14}$ although such instruments can suffer from drift if the mirror surface becomes contaminated. ${ }^{15}$ Commercial hygrometers based on 
thin-film capacitive sensors typically quote sensitivities of $\pm 1-3 \%$ for relative humidity measurement near room temperature, and with drift rates as low as one-half percent per year. ${ }^{16}$ Other methods include the use of infrared spectroscopy ${ }^{17-19}$ and surface-acoustic wave sensors. ${ }^{20}$

We chose to investigate the suitability of a commercial thin-film sensor for our supersaturation measurements, as these are available for very low cost and exhibit a high water vapor sensitivity with a very small sensor (thus requiring a low gas flow). To our knowledge, the use of these sensors for supersaturation measurements has not been well characterized in the published literature. For calibration of our instrument, we used saturated air at temperatures in the range $-30 \lesssim T \lesssim 10^{\circ} \mathrm{C}$, as this was sufficient for measuring supersaturation values for air temperatures in the range $-40 \leqq T$ $\lesssim 0{ }^{\circ} \mathrm{C}$, corresponding to our ice crystal growth experiments. Thin-film sensors are typically rated for substantially higher temperatures, but we did not examine the performance of our sensor above $10^{\circ} \mathrm{C}$.

After calibration, we found that the absolute accuracy of our sensor was somewhat unreliable, resulting in an uncertainty of $\pm 15 \%$ in the measured water vapor pressure. However, the instrument performed well in a differential mode, comparing supersaturated and saturated air at the same temperature. In the latter circumstance, we obtained supersaturation measurements that exhibited scatter at the $\pm 1.5 \%$ level, which is adequate for our ice crystal growth investigations.

\section{HARDWARE AND CALIBRATION}

The hygrometer used for these measurements was an inexpensive hand-held digital hygrometer, which is based on a thin-film capacitive sensor. ${ }^{21}$ The instrument accuracy is quoted as $\pm 3 \%$ in relative humidity, with operation over the temperature range $-20<T<50{ }^{\circ} \mathrm{C}$. Since the instrument is designed to operate under ambient conditions, it was necessary to remount the thin-film sensor and accompanying temperature sensor in a separate temperature-controlled housing, shown schematically in Fig. 1. The sensor housing was insulated from the outside environment, and its temperature was controlled using thermoelectric modules connected to a liquid-cooled back plate. The sensor temperature was typically maintained below ambient for our measurements. The sensor housing consisted of an aluminum box with a small sensor cavity (volume $<0.5 \mathrm{~cm}^{3}$ ) containing the hygrometer sensor and the thermistor temperature sensor that was part of the thermohygrometer unit. Wires between the sensors and controller were potted in place using low vapor-pressure epoxy, and connected to the hygrometer controller. Thin channels machined into the aluminum housing provided for air flow to and from the hygrometer sensor. The circuitous route traveled by the air ensured that the hygrometer sensor was adequately isolated from the room-temperature environment. In spite of the instrument's rated temperature range of -20 $<T<50^{\circ} \mathrm{C}$, we found that the built-in hygrometer controller would not indicate temperatures below $T=-16.6^{\circ} \mathrm{C}$. The hygrometer readings were quite sensible below this temperature, however, suggesting that only the controller display
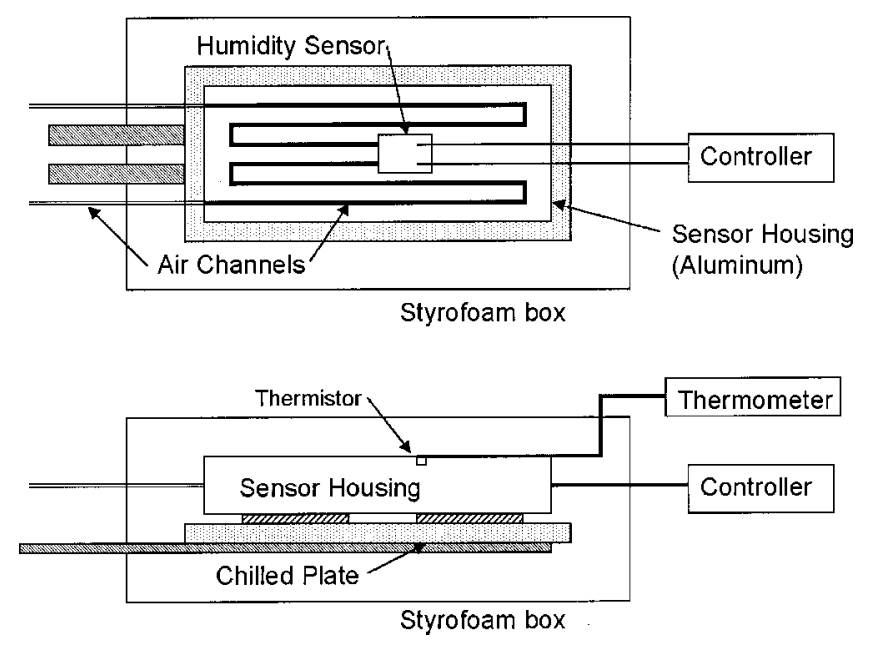

FIG. 1. Schematic top and side views of the temperature-controlled sensor housing, which contained the thin-film hygrometer sensor as well as thermistor temperature sensors. The 10-cm-long sensor housing was insulated from the outside environment, and its temperature was controlled using thermoelectric modules connected to a liquid-cooled back plate. Thin air channels machined into the aluminum housing provided for air flow to the sensor.

was erroneous. To ameliorate this problem, a separate calibrated thermistor temperature sensor, ${ }^{22}$ was attached to the aluminum box, and this external thermistor was used for all our measurements.

During operation of the hygrometer, sample air was moved through the housing using a small aquarium air pump operated in reverse as a vacuum pump, which pulled air though the sensor region at a flow rate of typically 0.02 $\ell /$ min replacing the air over the sensor approximately once per second. Air was transported from the sample region to the sensor using PE160 polyethylene tubing $(1.14 \mathrm{~mm}$ inner diameter).

Remounting the hygrometer sensor introduced some additional stray capacitance which changed the hygrometer readings somewhat. In particular, a typical controller reading of $45 \%$ relative humidity at room temperature became $60 \%$ after the sensor was moved. Thus, the hygrometer reading, $R$, nominally relative humidity, was treated simply as an independent variable related to the water vapor pressure and sensor temperature, which we then needed to calibrate.

Figure 2 shows a schematic of our calibration setup, which consisted principally of an insulated temperaturecontrolled $3.75 \ell$ jar containing a source of saturated air. Care was exercised in order to ensure that the jar reached a true equilibrium state, by making sure the water/ice in the jar was all at the lowest temperature point in the jar, and by waiting a time $t \gg L^{2} / D$, where $L$ is a typical jar dimension and $D \approx 2 \times 10^{-5} \mathrm{~m}^{2} / \mathrm{s}$ is the diffusion constant in air. Care was also taken that the air replaced into the jar did not substantially perturb the equilibrium condition. This was accomplished by drawing air into the jar through a $15 \mathrm{~m}$ length of Tygon tubing that was also held at the temperature of the jar. At the lowest jar temperatures the replacement air was first dried in a separate chamber, as shown in Fig. 2.

For a given calibration run, the air pump was turned on 


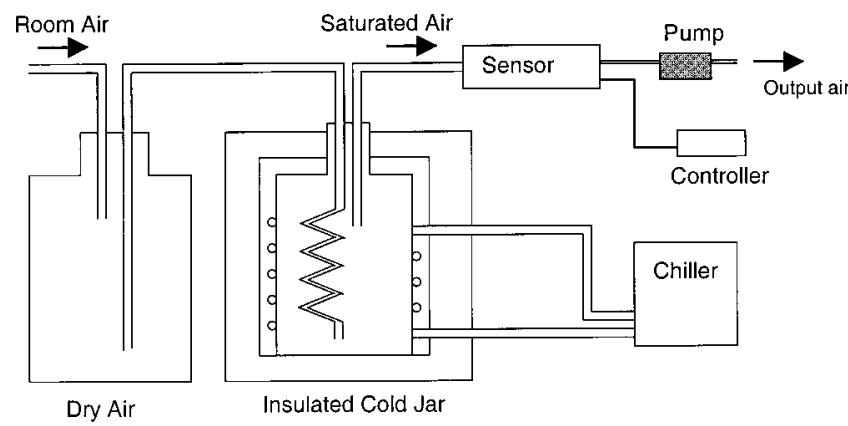

FIG. 2. Schematic drawing of the setup used to calibrate the humidity sensor. An insulated temperature-controlled $3.75 \ell$ jar provided a source of saturated air which was drawn through the sensor housing using a small vacuum pump. As described in the text, replacement air flowing into the cold jar was carefully conditioned so as not to disturb the water vapor equilibrium.

and the hygrometer reading was recorded as a function of time, with a typical result shown in Fig. 3. These data were fit to the functional form $R \rightarrow R_{0}+c_{0} \exp (-t / \tau)$, with fit constants $R_{0}, c_{0}$, and $\tau$. The time constant $\tau$ was found to show a nearly exponential dependence on sensor temperature, which is shown in Fig. 4. Although the time constant exceeded $2 \mathrm{~min}$ for $T=30^{\circ} \mathrm{C}$, the water vapor sensitivity of the device remained high down to this temperature. The time constant exhibited a minimum of about $4 \mathrm{~s}$, which may have been due to the response time of the digital controller. Waiting several time constants with the air pump on resulted in a stable hygrometer reading, although on occasion we observed some unexplained slow drift in the sensor reading.

\section{DATA ANALYSIS AND DISCUSSION}

Hygrometer data were acquired using saturated air at different source temperatures and different sensor temperatures, with the results shown in Fig. 5. The saturated water vapor pressure of the input air is a known function of the source temperature, which from the Clausius-Clapeyron relation is approximately $p_{\text {source }}\left(T_{\text {source }}\right)=p_{0} \exp \left(-T_{0} /\right.$

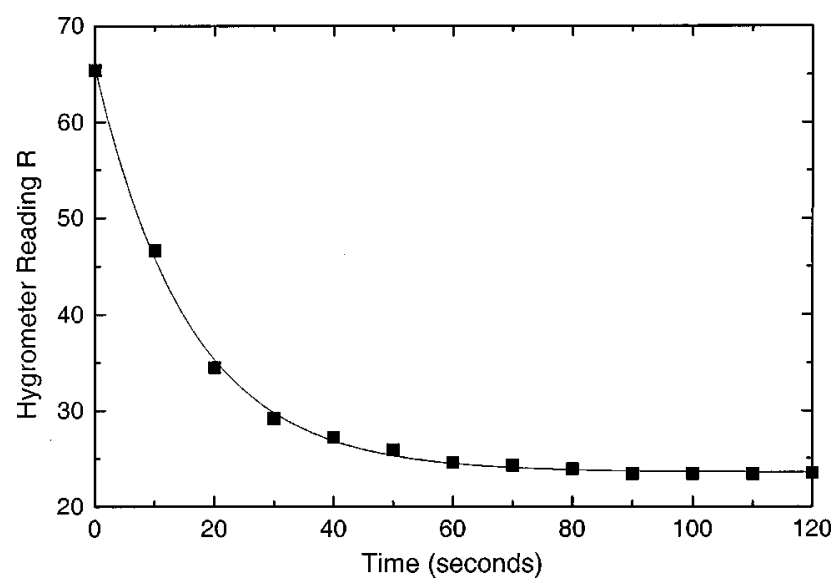

FIG. 3. Typical plot of relative humidity reading $R$ vs time, in this case for $\left(T_{\text {source }}, T_{\text {sens }}\right)=(242.2,284.2 \mathrm{~K})$. The fit line shows an exponential decay of the form $R=R_{0}+c_{0} \exp (-t / \tau)$.

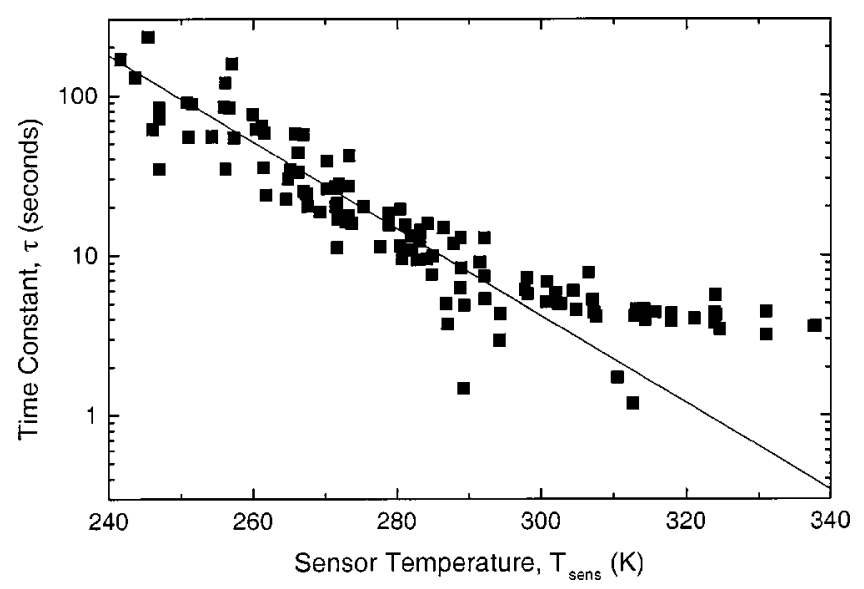

FIG. 4. Plot of the measured sensor time constant $\tau$ as a function of sensor temperature $T_{\text {sens }}$. The line shows an exponential dependence $\tau=5.8$ $\times 10^{8} \exp (-T / 16 \mathrm{~K}) \mathrm{s}$.

$T_{\text {source }}$ ), with $p_{0}=3.7 \times 10^{10} \mathrm{mbar}$ and $T_{0}=6150 \mathrm{~K} .{ }^{23}$ If the sensor calibration were ideal, then we would expect to measure a relative humidity of

$$
R H(\%)=100 \exp \left[-T_{0}\left(1 / T_{\text {source }}-1 / T_{\text {sens }}\right)\right]
$$

as a function of the source temperature $T_{\text {source }}$ and the sensor temperature $T_{\text {sens }}$. Since moving the hygrometer sensor changed its calibration, the measured values $R\left(T_{\text {source }}, T_{\text {sens }}\right)$ differed from this theoretical value. To derive a new calibration for the sensor, the different curves in Fig. 5 were fit to the functional form $R\left(T_{\text {source }}, T_{\text {sens }}\right) \rightarrow A\left(p_{\text {source }}\right) \exp \left(T_{1} /\right.$ $\left.T_{\text {sens }}\right)+B\left(p_{\text {source }}\right)$, where $A$ and $B$ are fit parameters. We chose to fix $T_{1}$ in order to reduce the number of fit parameters, and found that $T_{1}=T_{0}$ did not give the best results. We obtained quite good fits to all the data using a constant $T_{1}$ $=4000 \mathrm{~K}$, and this value was used for the subsequent analysis.

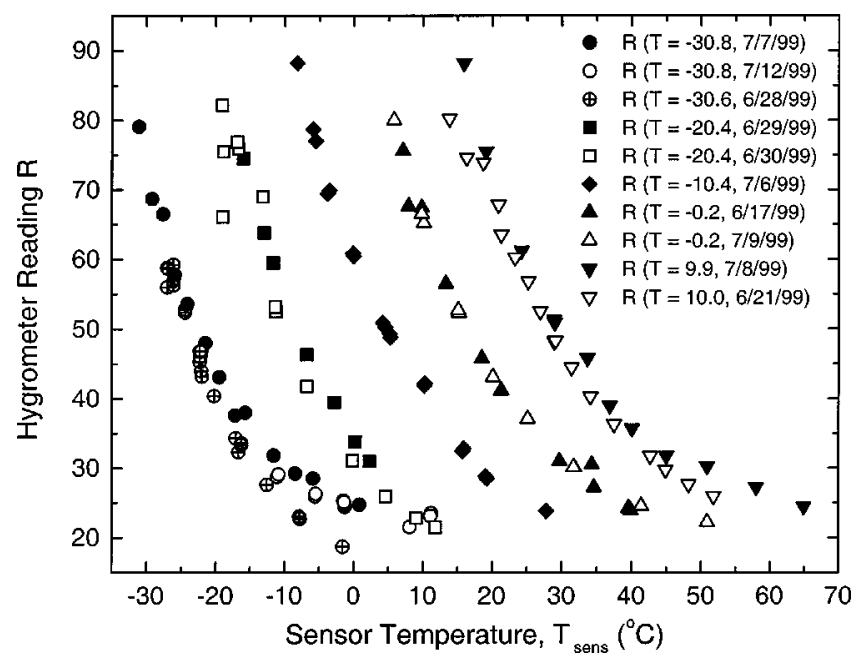

FIG. 5. Hygrometer reading $R$ vs sensor temperature $T_{\text {sens }}$, for saturated air at a variety of different source temperatures $T_{\text {source }}$. The general trend of the data is that expected from the Clausius-Clapeyron relation, although there are significant differences compared to the theoretically expected values. Note that runs on different days yielded somewhat different results, revealing some drift in the instrument. 


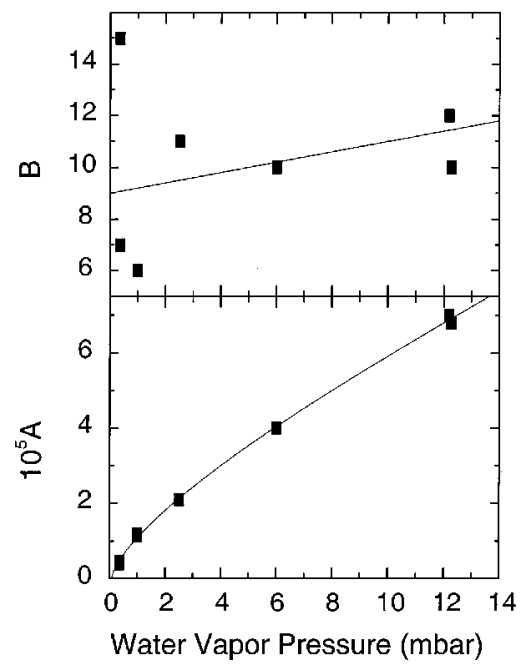

FIG. 6. Values of the fit coefficients $A$ and $B$ discussed in the text, as a function of water vapor pressure, obtained from the calibration data.

We then further fit the above fit parameters to the functional forms $A(p) \rightarrow C_{1}+C_{2} p^{1 / 2}+C_{3} p$ and $B(p) \rightarrow D_{1}$ $+D_{2} p$, where $p$ is taken to be in millibar, and obtained the best values $C_{i}=\left(-2.5 \times 10^{-6}, 1.06 \times 10^{-5}, 2.81 \times 10^{-6}\right)$ and $D_{i}=(9,0.2)$, as is shown in Fig. 6 . The calibrated hygrometer reading (in percent) is thus given by

$$
\begin{aligned}
R= & \left(C_{1}+C_{2} p^{1 / 2}+C_{3} p\right) e^{T_{1} / T_{\text {sens }}}+\left(D_{1}+D_{2} p\right) \\
= & \left(C_{3} e^{T_{1} / T_{\text {sens }}}+D_{2}\right) p+\left(C_{2} e^{T_{1} / T_{\text {sens }}}\right) p^{1 / 2} \\
& +\left(C_{1} e^{T_{1} / T_{\text {sens }}}+D_{1}\right)
\end{aligned}
$$

as a function of the sensor temperature and the vapor pressure of the input air. From this equation the water vapor pressure of the input air, $p$, can be determined from the measured $R$ and $T_{\text {sens }}$.

Going back to the data in Fig. 5, we calculated a value $p_{\text {calc }}$ for each $\left(R, T_{\text {sens }}\right)$ data point by solving the quadratic equation [Eq. (1)], which could then be compared with the known value of $p_{\text {source }}$. The difference was expressed as $\Delta p / p \equiv\left(p_{\text {source }}-p_{\text {calc }}\right) / p_{\text {source }}$, which is shown in Fig. 7 plotted against $R$ and against the temperature difference $T_{\text {source }}$ $-T_{\text {sens }}$.

We find from Fig. 7 that the uncertainty in the absolute vapor pressure measurements is about $10 \%-20 \%$, and the difference $\Delta p / p$ is higher for lower $R$ and for lower ( $\left.T_{\text {source }}-T_{\text {sens }}\right)$. It appears that the scatter in this calibration data arises from intrinsic drift in the capacitive thin-film sensor, and may be hysteretic in nature. Our laboratory conditions were not especially clean, which may have contributed to the sensor drift. We do not understand the origin of the observed drift, nor how to control it, and it thus effectively limits the applicability of this instrument for absolute measurements.

However, since the thin-film sensor was seen to have a high water vapor sensitivity down to quite low temperatures, we found that the instrument is well suited for differential measurements, even in the presence of some long-term drift. In particular, we obtained good results comparing supersaturated air with saturated air at the same temperature. For our application, we produce supersaturated conditions inside a nearly isothermal copper growth chamber $(46 \mathrm{~cm} /$ diameter and $61 \mathrm{~cm}$ length), and the formation of ice produces a boundary condition of zero supersaturation at the walls of the chamber. This boundary condition was further enforced (and protected from convection currents) by installing a length of Tygon tubing inside the chamber running around its circumference. Air drawn slowly from this length of tubing was thus at the ice saturation level for the temperature of the chamber walls, since the equilibrium time $\left(\approx L^{2} / D\right)$ is short inside the tubing.

A differential measurement was obtained by drawing supersaturated air from the center of the chamber, giving a point $\left(R_{\text {source }}, T_{\text {sens }}, T_{\text {source }}\right)$, and then immediately drawing saturated air to obtain a second point $\left(R_{\text {sat }}, T_{\text {sens }}, T_{\text {source }}\right)$. The water vapor pressure at the chamber center was then obtained by solving the equation
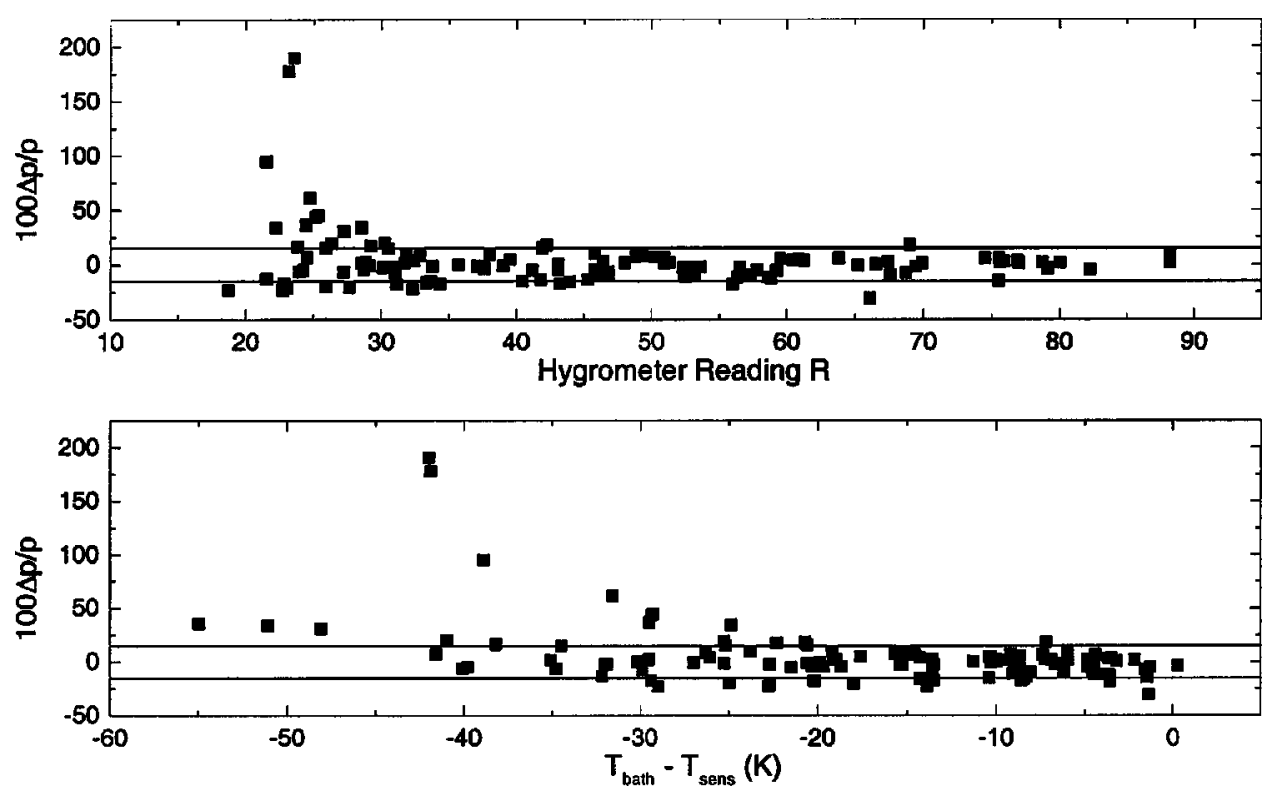

FIG. 7. Residual scatter in the calibration data, expressed as $\Delta p / p \equiv\left(p_{\text {source }}\right.$ $\left.-p_{\text {calc }}\right) / p_{\text {source }}$, and plotted vs hygrometer controller reading $R$ and vs temperature difference $T_{\text {source }}-T_{\text {sens }}$. Lines indicate $\pm 15 \%$ residual error. 


$$
\begin{aligned}
R_{\text {source }}-R_{\text {sat }}= & \left(C_{3} e^{T_{1} / T_{\text {sens }}}+D_{2}\right)\left(p_{\text {source }}-p_{\text {sat }}\right) \\
& +\left(C_{2} e^{T_{1} / T_{\text {sens }}}\right)\left(p_{\text {source }}^{1 / 2}-p_{\text {sat }}^{1 / 2}\right)
\end{aligned}
$$

for the vapor pressure $p_{\text {source }}$ using the fit coefficients $C_{i}$ and $D_{2}$ determined above.

We measured the residual uncertainty in the differential measurements by preparing stable supersaturated conditions in the growth chamber, and measuring the supersaturation as a function of time over several hours, using different sensor temperatures. The chamber temperature was maintained at $-5.0^{\circ} \mathrm{C}$, and the sensor temperature was in the range $0 \leqq T$ $\$ 25^{\circ} \mathrm{C}$, yielding hygrometer readings in the range $35 \%$ $\leq R \leq 80 \%$. From these data we inferred a mean supersaturation of approximately $10 \%$, with residual scatter of $\pm 1.5 \%$. Also the inferred supersaturation value was independent of the sensor temperature.

The techniques described above provide a simple and accurate measurement of the water vapor supersaturation of air in the temperature range $-40 \leqq T \leqq 0{ }^{\circ} \mathrm{C}$, which is suitable for further studies of the crystal growth of ice from the vapor phase. The procedure involves extracting a small volume of supersaturated air, warming it beyond the dew/frost point, and measuring the water vapor content using a commercial hygrometer based on a thin-film capacitive sensor. The time constant of the sensor was found to vary rapidly with sensor temperature, exceeding 2 min for $T<-30^{\circ} \mathrm{C}$, but the water vapor sensitivity of the device remained high down to this temperature. This technique has somewhat unreliable absolute accuracy, with an uncertainty of approximately $\pm 15 \%$ in the measured water vapor concentration. However, it performs well in a differential mode, producing supersaturation measurements with a residual uncertainty of $\pm 1.5 \%$.

\section{ACKNOWLEDGMENT}

The authors acknowledge financial support from the Summer Undergraduate Research Fellowship program of the California Institute of Technology.

${ }^{1}$ Ice crystal growth from vapor is reviewed by T. Kobayashi and T. Kuroda, in Morphology of Crystals, Part B, edited by I. Sunagawa (Terra Scientific, New York, 1987).

${ }^{2}$ K. G. Libbrecht, Phys. Rev. E 60, 1967 (1999).

${ }^{3}$ E. Yokoyama and T. Kuroda, Phys. Rev. A 41, 2038 (1990); E. Yokoyama, J. Cryst. Growth 128, 251 (1993).

${ }^{4}$ A. Yamashita, Kisho Kenkyu Noto, Met. Soc. Japan 123, 47 (1974).

${ }^{5}$ B. F. Ryan, E. R. Wishart, and D. E. Shaw, J. Atmos. Sci. 33, 842 (1976).

${ }^{6}$ T. Kuroda and R. Lacmann, J. Cryst. Growth 56, 189 (1982); T. Kuroda, ibid. 99, 83 (1990).

${ }^{7}$ J. Nelson and C. Knight, J. Atmos. Sci. 55, 1452 (1998).

${ }^{8}$ T. Kobayashi, Philos. Mag. 6, 1363 (1961).

${ }^{9}$ J. Hallett and B. J. Mason, Proc. R. Soc. London, Ser. A 247, 440 (1958);

N. Cho and J. Hallett, J. Cryst. Growth 69, 317 (1984).

${ }^{10}$ J. Nelson and C. A. Knight, J. Cryst. Growth 169, 795 (1996).

${ }^{11}$ M. Hanajima, Low Temp. Sci. A1, 53 (1944).

${ }^{12}$ T. Kobayashi, J. Meteorol. Soc. Jpn. 38, 231 (1960).

${ }^{13}$ General Eastern Instrument Products, http://www.geinet.com/Instrument/ InstHome.htm, Models E4, M2, M3, and M4 (1999).

${ }^{14}$ Buck Research, http://www.buck-research.com/products.htm, Models CR-1, CR-2, and 1011B (1999).

${ }^{15}$ M. Hoenk, private communication (1999).

${ }^{16}$ Vaisala Corp., http://www.vaisala.com/inc/ssdcat (1999).

${ }^{17}$ W. Chen, C. Przygodzki, H. Delbarre, P. Peze, J. Burie, and D. Boucher, Infrared Phys. Technol. 39, 415 (1998).

${ }^{18}$ T. A. Cerni, J. Atm. Oceanic Tech. 11, 445 (1994).

${ }^{19}$ M. P. Arroyo and R. K. Hanson, Appl. Opt. 32, 6104 (1993).

${ }^{20} \mathrm{M}$. Hoenk, private communication, and http://mishkin.jpl.nasa.gov/ spacemicro/MWS_PAPER (1999).

${ }^{21}$ Mannix Corp., Thermo-hygrometer Model \#LAM880D/8703.

${ }^{22}$ YSI Incorporated (Yellow Springs, OH 45387), Precision Thermistor Model \#44033.

${ }^{23}$ The constants $p_{0}$ and $T_{0}$ were obtained by fitting the Clausius-Clapeyron relation to data for the saturated water vapor pressure of ice. 